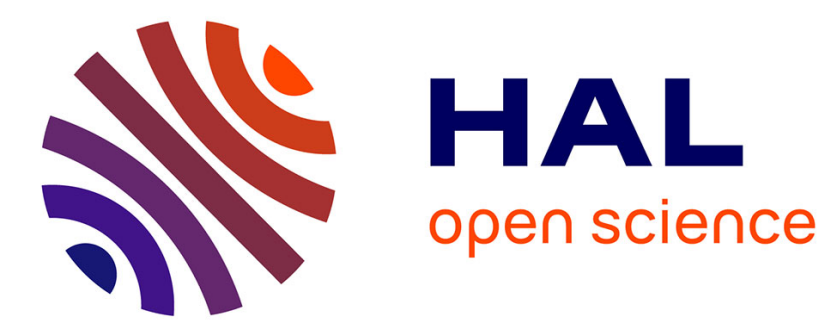

\title{
Super-resolution with continuous scan shift
}

\author{
François Orieux, Thomas Rodet, Jean-François Giovannelli
}

\section{To cite this version:}

François Orieux, Thomas Rodet, Jean-François Giovannelli. Super-resolution with continuous scan shift. 16th IEEE International Conference on Image Processing (ICIP), Nov 2009, Le caire, Egypt. 10.1109/ICIP.2009.5413718 . hal-00416568

\section{HAL Id: hal-00416568 \\ https://hal.science/hal-00416568}

Submitted on 5 Mar 2020

HAL is a multi-disciplinary open access archive for the deposit and dissemination of scientific research documents, whether they are published or not. The documents may come from teaching and research institutions in France or abroad, or from public or private research centers.
L'archive ouverte pluridisciplinaire HAL, est destinée au dépôt et à la diffusion de documents scientifiques de niveau recherche, publiés ou non, émanant des établissements d'enseignement et de recherche français ou étrangers, des laboratoires publics ou privés. 


\title{
SUPER-RESOLUTION WITH CONTINUOUS SCAN SHIFT
}

\author{
François Orieux, Thomas Rodet and Jean-François Giovannelli \\ Laboratoire des Signaux et Systèmes (CNRS - SUPELEC - Univ. Paris-Sud 11) \\ SUPELEC, Plateau de Moulon, 3 rue Joliot-Curie - 91192 Gif-sur-Yvette Cedex, France. \\ e-mail: orieux, rodet, giova@lss.supelec.fr
}

\begin{abstract}
Super-resolution methods aimed to restore the spectrum of an original image above the half sampling frequency. The restoration problem is generally viewed as an inverse problem and a lot of research focus on the inversion approach. In this paper the question of super-resolution is addressed with only the forward model point of view. With the addition of the time dimension in an specific case of continuous shift scanning we obtain explicit expression for the forward model and the data spectrum. We show with a new, simple and rigorous formalism that super-resolution can be done because the whole set of data is less aliased than a single image of the scene. Moreover, limits, conditions and performance questions are also addressed as well as perspectives on sampling conditions.
\end{abstract}

Index Terms - Image restoration, image sampling, super-resolution.

\section{INTRODUCTION}

Super-resolution image restoration methods intend to restore frequency of an image above the half sampling frequency $1 / 2 L$ of a sensor [1] with $L$ as pixel size. To achieve this, the methods usually consider a collection of noisy blurred and under-sampled images of the same scene. The small shift inferior to a pixel size [2] between the images allow to restore higher frequencies.

The problem to restore a unique single image from this data set is generally viewed as an inversion problem with a forward model that take into account the motion and other sensor effects. A lot of research is done to produce new restoration algorithms that combine better motion estimation [3], new image prior field or better forward model [4] (such as transformations other than translations) for example.

In this paper the de-aliasing is only addressed from the forward point of view rather than the inversion point of view. The shift is considered to be continuous in one direction, encountered in spatial imaging, satellite imaging or robotics. In this shift case and with the addition of the time dimension in the formalism, we obtain an explicit expression of the data spectrum. We show easily with this new formalism that, while the image spectrum is aliased, the introduction of motion in time produce data that are less aliased: any method that properly take into account this information naturally achieve super-resolution.

\section{FORWARD MODEL}

This section describes the forward model. A signal $\phi(x) \in$ $L_{2}: \mathbb{R} \rightarrow \mathbb{R}$ is firstly shifted and secondly integrated and sampled by a sensor. Spectrum of the data is derived.

\subsection{The shift}

The continuously shifted signal $\phi_{s}$ is written as a function of time

$$
\phi_{s}(x, t)=\phi(x-p(t))
$$

where $p(t)$ corresponds to the protocol describing the evolution of the translation. The case of the continuous spatial translation with constant rate is $p(t)=v t+x_{0}$ where $\left(v, x_{0}\right)$ are the scan speed and the origin point at the beginning of the acquisition. The Fourier transform of the shifted signal in the spatial dimension is

$$
\stackrel{\circ}{\phi}_{s}\left(f_{x}, t\right)=\stackrel{\circ}{\phi}\left(f_{x}\right) e^{-2 j \pi f_{x} p(t)} .
$$

It is the spectrum of the original signal with a phase that depends on the shift protocol. It is interesting to note that it is only the additional phase term that depends on time. The importance of this point will appears later.

\subsection{Spatial and temporal sampling}

The shifted signal is spatially and temporally integrated by the sensor. Each sensor element $l$, centered in coordinate $x_{l}$, has a size $L$. The optical convolution and sensor response can be sum up by $h(x)$. In addition the signal is temporally sampled in time with a period $T$. When the sensors are regularly spaced in space and time, it is generally more convenient to described this acquisition with the convolution of the signal by $h(x)$ followed by a multiplication with a Dirac comb. Then the sensor acquisition is written

$$
y(x, t)=\Delta_{L}(t) \Delta_{T}(t) \int_{\mathbb{R}} \phi_{s}\left(x^{\prime}, t\right) h\left(x^{\prime}-x\right) \mathrm{d} x^{\prime}
$$


where $\Delta_{L}(x)=\sum_{l} \delta(x-l L)$ and $\Delta_{T}(t)=\sum_{n} \delta(t-n T)$ and $\delta$ the Dirac distribution. However this model potentially supposes that an infinite number of samples are available. Then the field of view of the sensor $\mathcal{L}$ as well as the total time of observation $\mathcal{T}$ are introduced with a rectangular function $\mathbb{1}$

$$
\begin{array}{r}
y(x, t)=\mathbb{1}_{\mathcal{L}}(x) \mathbb{1}_{\mathcal{T}}(t) \Delta_{L}(x) \Delta_{T}(t) \\
\int_{\mathbb{R}} \phi_{s}\left(x^{\prime}, t\right) h\left(x^{\prime}-x\right) \mathrm{d} x^{\prime}
\end{array}
$$

where $\mathbb{1}_{\mathcal{L}}(x)=1$ if $-\mathcal{L} / 2 \leq x \leq \mathcal{L} / 2,0$ elsewhere. It is important to notice that the data are described in continuous formulation even with the sampling step.

\subsection{The data spectrum}

Super-resolution method aimed to restore the frequencies above the sampling frequency $1 / 2 L$ and reduce aliasing. To understand how the introduction of several spatial acquisition with smalls shift between them allow to restore this frequencies, the data Fourier transform $\stackrel{\circ}{y}$ in spatial $f_{x}$ and time $f$ frequency will be helpful

$$
\stackrel{\circ}{y}\left(f_{x}, f\right)=\iint_{\mathbb{R}^{2}} y(x, t) e^{-2 j \pi\left(f_{x} x+f t\right)} \mathrm{d} x \mathrm{~d} t .
$$

Since data is the result of convolution and multiplication, the spectrum in spatial frequencies can be rewrites as a convolution of $\dot{\circ}_{s} \stackrel{\circ}{h}$ with $\stackrel{\circ}{1}_{\mathcal{L}} \stackrel{\circ}{\Delta}_{1 / L}$ in the $f_{x}$ axis

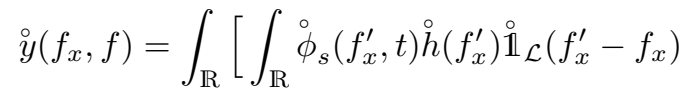

$$
\begin{aligned}
& \left.\Delta_{1 / L}\left(f_{x}^{\prime}-f_{x}\right) \mathrm{d} f_{x}^{\prime}\right] \mathbb{1}_{\mathcal{T}}(t) \Delta_{T}(t) e^{-2 j \pi f t} \mathrm{~d} t .
\end{aligned}
$$

With the expression (2), the data spectrum $\stackrel{\circ}{y}\left(f_{x}, f\right)$ become

$$
\int_{\mathbb{R}} \stackrel{\circ}{y}_{s}\left(f_{x}^{\prime}\right) \int_{\mathbb{R}} \mathbb{1}_{\mathcal{T}}(t) \Delta_{T}(t) e^{-2 j \pi f_{x}^{\prime} p(t)} e^{-2 j \pi f t} \mathrm{~d} t \mathrm{~d} f_{x}^{\prime}
$$

where

$$
\stackrel{\circ}{y}_{s}\left(f_{x}^{\prime}\right)=\stackrel{\circ}{\phi}\left(f_{x}^{\prime}\right) \stackrel{\circ}{h}\left(f_{x}^{\prime}\right) \stackrel{\circ}{1}_{\mathcal{L}}\left(f_{x}^{\prime}-f_{x}\right) \Delta_{1 / L}\left(f_{x}^{\prime}-f_{x}\right)
$$

is the spectrum in spatial frequencies and doesn't depends on time. Moreover, the integration on time in (7) doesn't depends on the signal.

Now we can use the expression of the particular shift protocol $p(t)=v t+c$. Starting from (7) the integral on time is a Fourier transform and is explicit

$$
\begin{aligned}
& \int_{\mathbb{R}} \mathbb{1}_{\mathcal{T}}(t) \Delta_{T}(t) e^{-2 j \pi\left(f_{x}^{\prime} v+f\right) t} \mathrm{~d} t= \\
& \sum_{n} \mathcal{T} \operatorname{sinc}\left[\mathcal{T}\left(f_{x}^{\prime} v+f+\frac{n}{T}\right)\right]
\end{aligned}
$$

where $\operatorname{sinc}(x)=\sin (\pi x) / \pi x$. It is the $1 / T$ periodic spectrum of a squared window with dual variable $f_{x}^{\prime} v+f$.

Finally the data spectrum can be derived

$$
\begin{aligned}
& \stackrel{\circ}{y}\left(f_{x}, f\right)=\sum_{l, n} \int_{\mathbb{R}} \stackrel{\circ}{\phi}\left(f_{x}^{\prime}\right) \stackrel{\circ}{h}\left(f_{x}^{\prime}\right) e^{-2 j \pi f_{x}^{\prime} x_{0}} \\
& \mathcal{T}_{\text {sinc }}\left[\mathcal{T}\left(f_{x}^{\prime} v+f+\frac{n}{T}\right)\right] \\
& \stackrel{\circ}{\mathbb{L}}_{\mathcal{L}}\left(f_{x}^{\prime}-f_{x}+\frac{l}{L}\right) \mathrm{d} f_{x}^{\prime} .
\end{aligned}
$$

In order to have a simpler expression, easier to analyse, the spatial field of view is considered infinite. It's Fourier transform is a Dirac $\stackrel{\circ}{\mathbb{1}}_{\mathcal{L}}\left(f_{x}\right)=\delta\left(f_{x}\right)$ and the simplified spectrum writes

$$
\begin{aligned}
\stackrel{\circ}{y}\left(f_{x}, f\right)=\sum_{l, n} \stackrel{\circ}{\phi}\left(f_{x}^{l}\right) \stackrel{\circ}{h}\left(f_{x}^{l}\right) e^{-2 j \pi f_{x}^{l} x_{0}} \\
\mathcal{T}_{\text {sinc }}\left[\mathcal{T}\left(f_{x}^{l} v+f+\frac{n}{T}\right)\right]
\end{aligned}
$$

with $f_{x}^{l}=f_{x}-l / L$. It is a periodic spectrum in both axis with the spectrum of the original signal $\stackrel{\circ}{\phi}$, the transfer function $\stackrel{\circ}{h}$ and the transform due to time.

\section{DATA SPECTRUM DISCUSSIONS}

In this section we discuss (10) that is the spectrum of data obtained with a continuous shift of the signal in one direction:

- First, their is a sum over the index $l$ and $n$. This index represent respectively the spatial and temporal replication because of sampling.

- Second, the signal spectrum $\stackrel{\circ}{\phi}$ and the transfer function $\stackrel{\circ}{h}$ depend only on the spatial frequency $f_{x}$, not on $f$.

- Then, a sinc function has replaced the phase of the shift. This sinc function depends on the two frequencies $f_{x}$ and $f$.

\subsection{Analyze}

\subsubsection{Introduction of the time}

In comparison to a sampled filtered signal spectrum, the main difference point in the shifted signal spectrum is the sinc function. Firstly, this is the only part that depend on $f$. The original signal spectrum and the transfer function does not depend on $f$. Secondly, it depends on the shift protocol parameters $(\mathcal{T}, v)$.

In other word, the shift of the signal during the time has introduced a second dimension $f$ in the data spectrum. The original signal spectrum does not depends on it but a supplementary sinc term, that depends on the shift parameter has been added and apply a transformation on the signal spectrum. 


\subsubsection{Influence of the time}

For more clarity of the description, the problem is reduced to one period $n=0$ in $f$ axis. Then

$$
\stackrel{\circ}{y}\left(f_{x}, f\right)=\sum_{l} \stackrel{\circ}{\phi}_{f}\left(f_{x}^{l}\right) \mathcal{T} \operatorname{sinc}\left[\mathcal{T}\left(f_{x}^{l} v+f\right)\right]
$$

with the filtered signal $\dot{\circ}_{f}\left(f_{x}\right)=\stackrel{\circ}{\phi}\left(f_{x}^{l}\right) \stackrel{\circ}{h}\left(f_{x}^{l}\right) e^{-2 j \pi f_{x}^{l} x_{0}}$. If the sinc function where not present we retrieve the periodic spectrum $\sum_{l}{ }^{\circ} \phi_{f}\left(f_{x}^{l}\right)$ with aliased frequency if $L$ is not sufficiently small.

To illustrate the influence of the time, the Fig. 1 shows the data spectrum. The Fig. 1(a) is the periodic signal spectrum $\sum_{l}{ }^{\circ} \phi_{f}\left(f_{x}^{l}\right)$ expressed in $f_{x}$ and $f$. The Fig. 1(b) is the periodic sinc part $\sum_{l} \mathcal{T}$ sinc $\left[\mathcal{T}\left(f_{x}^{l} v+f\right)\right]$. On Fig. 1(c), each period of index $l$ of the signal spectrum has been multiplied with a sinc function that depends also on $l$ like

$$
\operatorname{sinc}\left[\mathcal{T}\left(\left(f_{x}-\frac{l}{L}\right) v+f\right)\right] .
$$

The maximum of each sinc function is determined by the line

$$
\left(f_{x}-l / L\right) v+f=0 \quad \text { or } \quad f=-f_{x} v+l v / L .
$$

As illustrated on Fig. 1(b), the origin of those lines (or the sinc functions) on axis $f$ depends on the index $l$ but also on $v$ and $L$. The inclination of the line (or the sinc function) depends only on $v$.

\subsection{Super-resolution}

Since each period $l$ of the signal spectrum Fig. 1(a) is multiplied with the corresponding sinc function $l$ Fig. 1(b), each period is present on different place in the Fourier plane as illustrated by Fig. 1(c). The support of the sinc function is infinite but it's energy can be neglected after some level. Therefor each period can be considered separated: there is no more aliasing or aliasing is greatly reduced.

This simple equation (10), illustrated on Fig. 1, explains why, when there is a continues shift in one direction, the restoration by super-resolution method is possible. Effectively, in the data spectrum there is less aliasing. Then, if the method uses properly this data to restore the original signal, it is possible to restore frequency above the sampling frequency.

\subsection{Limits and conditions}

This specific case allows to obtain a well and precise description with explicit equation (10). Then it is possible to obtain conditions or criteria based on the parameters of the shift protocol that assure that there is no aliasing in data. In addition some limits behaviour and conditions can be derived that depend also on the shift parameters.

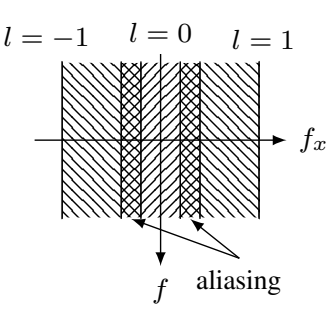

(a)

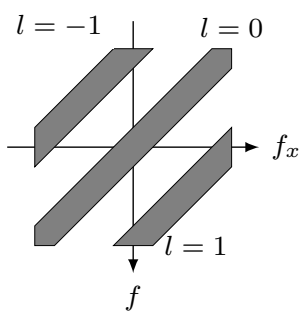

(b)

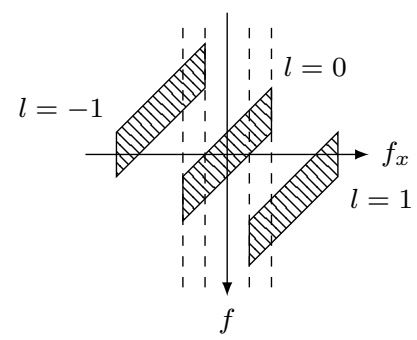

(c)

Fig. 1. The support of the spectrum. The Fig. (a) is the sampled filtered signal spectrum support. This spectrum is defined only on $f_{x}$ and is extended along the $f$ axis. The Fig. (b) is the support of the central lobe of the sinc functions. The data spectrum is illustrated on Fig. (c). It is the sum of the product of each $l$-replication of the signal with the corresponding $l$-replication of the sinc function described by (11).

\subsubsection{Example of condition}

The support of the sinc functions are infinite. As a consequence the separation is not perfect and there is always aliasing. However, as a criterion the support of the first $N$ zero of each sinc function is used as a limit beyond which there is no superposition. In these case the influence support of each sinc is between the two lines

$$
f=f_{x} v+\frac{l v}{L} \pm \frac{N \pi}{\mathcal{T}}
$$

Then two periods with index $l$ and $l+1$ are separated if

$$
f_{x} v+\frac{l v}{L}+\frac{N \pi}{\mathcal{T}}<f_{x} v+\frac{(l+1) v}{T}-\frac{N \pi}{\mathcal{T}} .
$$

if and only if

$$
v \mathcal{T}>2 \pi N L .
$$

Even if $1 / L$ is smaller than the Nyquist frequency, if the two parameters $v$ and $\mathcal{T}$ are set to assure this inequality, the data has not aliased with respect to this condition.

\subsubsection{Limits of the scan speed $v$}

If the scan speed $v=0$, all the maxima are on the line $f=0$ as described by (13). The sensor doesn't move and no more 
super-resolution is possible. The obtained data spectrum is

$$
\stackrel{\circ}{y}\left(f_{x}, f\right)=\mathcal{T} \sum_{l} \stackrel{\circ}{\phi}_{f}\left(f_{x}^{l}\right)
$$

the image spectrum amplified by $\mathcal{T}$ : the observation in time improve the SNR of data. If the scan speed is $v=+\infty$, the maximum of each sync is on the line $f_{x}=-l / T$ and

$$
\lim _{v \rightarrow+\infty} \operatorname{sinc}\left[\mathcal{T}\left(f_{x}^{l} v+f\right)\right]=0 .
$$

All the energy is lost, the sensor doesn't have the time to integrate the signal.

If $v T=q L$ with $q \in \mathbb{N}$ (the distance in one period of time is a multiple of the pixel size), with the periodization in $f$ axis, the maximum lines are

$$
f=-f_{x} v+(q l-n) / T .
$$

No super-resolution is possible because there at least two lines $(l, n)$ and $\left(l^{\prime}, n^{\prime}\right)$ that are identical: the period are, again, superimposed.

\subsubsection{Limits of the temporal sampling period $T$}

If the sampling period is $T=0$ there is a perfect sampling (the continuous signal is acquired). All the maximum line, except for $l=0$, go to infinite. The period are perfectly separated and super-resolution restoration are potentially perfect.

If the sampling period $T=+\infty$, all the maximum lines are $f=f_{x} v$ for all $l$. All the sinc function are overcome. No more super-resolution is possible since, at most, only one temporal sample as been acquired.

\subsubsection{Limits of the total observation time $\mathcal{T}$}

If the total observation time $\mathcal{T}=0$ there is no observation at all and the data spectrum is null as described by (10). If $\mathcal{T}=+\infty$ the integral over $t$ in (9) writes

$$
\int_{\mathbb{R}} \Delta_{T}(t) e^{-2 j \pi\left(f_{x}^{\prime} v+f\right) t} \mathrm{~d} t=\sum_{n} \delta\left(f_{x}^{\prime} v+f+\frac{n}{T}\right) .
$$

All the sinc function become Dirac distributions and the period are perfectly separated.

\section{EXTENSION TO IMAGE}

The extension to image is straightforward and is based on the addition of a second dimension. The expression of the data spectrum is more complex but the structure still the same

$$
\begin{aligned}
& \stackrel{\circ}{y}\left(f_{\alpha}, f_{\beta}, f\right)=\sum_{l, m, n} \stackrel{\circ}{\phi}\left(f_{\alpha}^{l}, f_{\beta}^{m}\right) \stackrel{\circ}{h}\left(f_{\alpha}^{l}, f_{\beta}^{m}\right) e^{-2 j \pi f_{\alpha}^{l} c_{\alpha}} \\
& e^{-2 j \pi f_{\beta}^{m} c_{\beta}} \mathcal{T}_{\operatorname{sinc}}\left[\mathcal{T}\left(f_{\alpha}^{l} v_{\alpha}+f_{\beta}^{m} v_{\beta}+f+\frac{n}{T}\right)\right] .
\end{aligned}
$$

In this case, the previous condition (16) is written: two periods with index $(l, m)$ and $\left(l^{\prime}, m^{\prime}\right)$, respectively, are not aliased if

$$
\left(\left(l-l^{\prime}\right) v_{\alpha}+\left(m-m^{\prime}\right) v_{\beta}\right) \mathcal{T}<2 \pi N L .
$$

\section{CONCLUSIONS}

This paper describe and explain, with a description in Fourier space of the forward model, how super-resolution is possible. The computation of the data spectrum is done in a special case of a continuous shift in one direction during the whole observation. Thanks to the addition of the time dimension in the formalism, we show that restoration of image frequencies above the spatial sampling frequency is possible because the data are less aliased. Since this property is about data, it is available to any processing method that take into account the time. The formalism allow also to address some questions about the limits and performances of super-resolution. Future work can focus on the consequence of the finite field of view in the limits and conditions, finer analyse of the $f$ axis periodization, and more general motion protocol.

\section{REFERENCES}

[1] S. C. Park, M. K. Park, and M. G. Kang, "Superresolution image reconstruction: a technical overview," IEEE Trans. Signal Processing Mag., pp. 21-36, May 2003.

[2] M. Elad and A. Feuer, "Restoration of a single superresolution image from several blurred, noisy, and undersampled measured images," IEEE Trans. Image Processing, vol. 6, no. 12, pp. 1646-1658, December 1997.

[3] M. Protter, M. Elad, H. Takeda, and P. Milanfar, "Generalizing the nonlocal-means to super-resolution reconstruction," IEEE Trans. Image Processing, vol. 18, pp. 36-51, January 2009.

[4] Gilles Rochefort, Frédéric Champagnat, Guy Le Besnerais, and Jean-François Giovannelli, "An improved observation model for super-resolution under affine motion," IEEE Trans. Image Processing, vol. 15, no. 11, pp. 3325-3337, November 2006. 